\title{
Design and Implementation of Sign Language Interpretation System and Wireless Enabled Wheel Chair
}

\author{
S. Dhanalakshmi \\ Assistant Professor \\ Department of Electronics and \\ Communication System \\ Coimbatore Institute of Technology, \\ Coimbatore
}

\author{
N. Jayakumar \\ Final year \\ Department of Electronics and \\ Communication System \\ Coimbatore Institute of Technology, \\ Coimbatore
}

\author{
S. MithunKumar \\ Final year \\ Department of Electronics and \\ Communication System \\ Coimbatore Institute of Technology, \\ Coimbatore
}

\begin{abstract}
Sign language is used by speech and hearing-impaired persons to convey their message to other people. Often this sign language is not understood by everyone, which produces a communication gap. To bridge this gap, smart gloves are introduced. The system is based on sensor gloves which convert the sign language to speech. The gloves read the motion and orientation of both hands through Accelerometer and Flex sensors sewed within. All the sensor data are processed and multiplexed wirelessly through Bluetooth. When no sign is made, the system has the capability to switch to its sleeping mode which enables it to save the power. The system design uses a simple algorithm that directly maps gestures via lookup process, thus avoiding complex computations. The aim is to develop a portable, reliable and an inexpensive system. Therefore, the system uses less expensive electronics which are easily available in the local market, making the system affordable for everyone. In this proposed work the Arduino microcontroller, accelerometer, and Bluetooth are used. Two main contributions are presented in this work. Firstly, it is shown that the wheel chair can be controlled with hand-gestures according to the movement and position of the hand. Secondly, the proposed wheel chair system is further extended to be controlled by an android based mobile application.
\end{abstract}

\section{Keywords}

Flex sensor, Android, Accelerometer, Bluetooth, Arduino, Smart car, smart gloves

\section{INTRODUCTION}

Deafness or hearing-impairment is the loss of ability to hear normally whether permanent or unstable. It can be caused by environmental and genetic factors as well. According to WHO (World Health Organization) estimates, 360 million people worldwide are suffering from hearing loss and this could increase to 900 million by 2025 . The disabilities in household members are considered as stigma and threat to family's social status. In a study conducted, it was found that hearing impairment students have a hard time adjusting with the other hearing classmates. Communication problems have caused them isolation from the society. Deaf use sign language as a medium of communication with the world. Sign language is a complex set of gestures which includes facial expressions, hand movements, bending of fingers and arms position. Most of the signs are made from hand movements. Sensing these motions, many systems for sign language translation have been made. The paper is organized in such a way that Section II provides the reviewed literature, Section III introduces the proposed system, Section IV gives the methodology of the system, Section V gives the experimental results and Section
VI provides the concluding remarks along with the future application.

\section{LITERATURE REVIEW}

B. G. Lee et.al., (2018) collected gesture sample data using sensor gloves which has five bending sensors and then processed and optimized using PSO algorithm. [1] Inertial motion sensing glove was designed to detect movement of the entire limb. 3 axis accelerometer sensors (ADXL) were used on each finger. Vision technique is based on images processing Non-Vision based on Flex, Motion Sensors and Pressure Sensors.

Chun Zhu et.al., (2017) proposes the gesture recognition system using sensor gloves. Their applications are vast ranging from Biomedical, Virtual Reality, Robotics, Design and Manufacturing to Sign language translating. A survey paper for the applications of sensor gloves and their classification is presented [2]. A gesture recognition-based Human-Computer Interaction control system is developed via Lab VIEW to control a robot wirelessly through hand gestures. An improved PSO-SVM (Particle Swarm Optimization) classification algorithm of hand-gesture recognition is proposed to increase the precision and efficiency of gesture recognition.

Wang Jingqiu1 et.al., (2018) proposes a precise hand gesture modeling using the sensor gloves with RGB camera for vision-based gesture recognition. The approaches of Hidden Markov Model (HMM) and parallel HMM were used for sensor fusion modeling.

S.V.Matiwade et.al., (2017) proposes another sensorcomputer based hybrid inertial sensor glove system. The system tracks hand movements using inertial measurements and the real-time data is fed to a machine learning algorithm for gesture recognition based on LDA (Linear Discriminant Analysis) to reduce classification complexities [3]. The data was captured using a camera mounted on the hat to give the vision-based system a mobility factor and accelerometers are placed on the wristbands to orient the wheel chair to the required direction. Accelerometer data provides additional information to the camera captured data. However, the results were too noisy because of the webcam used.

Mikhail G. Grif, et.al., (2018) proposes hand gestures Recognition, Image processing skeletal Tracking and Gesture Detection system. This is vison-based approach when a camera is used to record the hand movements and the video is partitioned into frame. For each frame set, features are extracted. SURF (Speed Up Robust Feature) is used for detection and feature recognition [4]. This system recognizes 26 alphabets and some gesture actions using image processing 
and it is implemented in Python environment.

Hardik Rewari et.al., (2018) proposes SLI algorithm to calculate the resistance variation in the flex sensor [5]. Based on the resistance variation the corresponding sign language is interpreted. With the help of various components like flex sensors, MPU6050, HC-05, SD-Card module etc., replication of few signs from the Indian Sign Language was interpreted.

Andrews Samraj et.al., (2017) proposes robust sign language system for the usage of the disabled in the case of communication and common people also can use the security features [6]. Data Glove and PPG fusion helps in growing the Euclidean distance up to 10 times higher than the normal data glove usage in security. The simple Euclidean distance calculations are found enough to establish the system without much difficult mathematical modeling or complex classifiers. This proves that this system can be implemented without much computational complexity.

Soniya D et.al., (2016) proposes four basic requirements for self-governing operations for people with motor-impairments are Mobility, Ambient control, Health monitoring and emergency handling stands vital for transitioning to living independently [7]. Blending the four above enlisted fundamental requirements and the result presented in the paper will aid with great possible in becoming solid solution for real life problems of the motor impaired.

Vishal P. Bhujbal et.al., (2018) proposes a smart hand sign interpretation system using a smart glove to reduce the communication gap between speech impaired people and the normal people. This wearable system utilizes five flexsensors, 3-axis accelerometer, one Bluetooth module and $16 * 2$ LCD display [8]. Data matching was done internally and if a matched word is not found, then its equivalent word is sent to LCD and Android app. Android mobile app can convert this into voice. So this system has an ability to convert sign language into a voice in a very simple way.

\section{PROPOSED METHOD}

\subsection{Sign Language}

Sign language system is a mode of communication between deaf and dumb people. Sign language emerges and evolves naturally for hearing impaired community. Sign language communication has non-manual signs involve fingers, hands, arms and non-manual signs involve face, head, eyes and body. The normal people who can talk and hear properly and who have the knowledge of sign language can communicate with deaf and dumb people, but the people who does not have the knowledge on sign language cannot communicate to deaf and dumb people. Sign language to text and speech conversion will be more useful for such impaired people to communicate with normal people.

\subsection{Types of Sign Language \\ Indian Sign Language (ISL)}

American Sign Language (ASL)

Indian Sign Language uses both hands to represent each alphabet and gesture and these alphabets are derived from British Sign language and French sign language [8]. American Sign Language is the most widely used because most of the signs in ASL are single handed and thus, the complexity is less and also ASL has strong database that is available for use Shows in Fig 1.

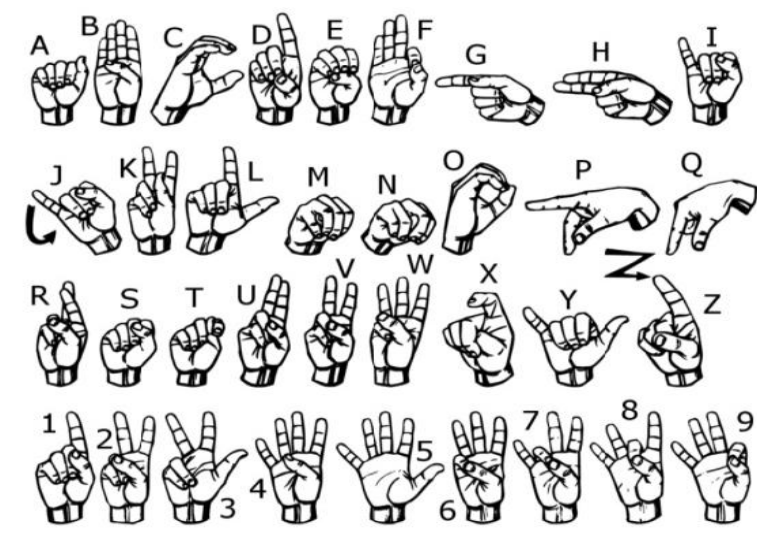

Fig.1: Sign Language

\subsection{Hardware design}

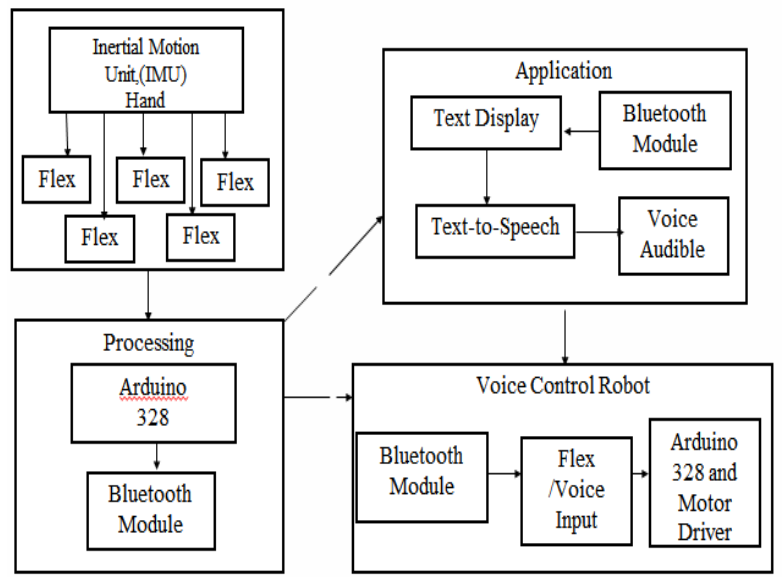

Fig.2: Block diagram of proposed model

The block diagram of the proposed system is shown in Fig.2 It comprises of following four major modules:

- Data Acquisition Module

- $\quad$ Signal Conditioning and Processing Module

- Data Transmission and Reception Module

- $\quad$ Audio Module

\section{A. Data Acquisition:}

The system comprises of two hand sensor gloves which will be able to translate both single and double-handed gestures. The gloves will track angular position, rotation of hands and bending of fingers. These motions are read through the use of multiple flex sensors stitched within the gloves. The resistance of sensor changes as the fingers are bent. The bending of fingers creates variations in resistances and these variations are transformed into voltages by using flex sensors in voltage-divider configuration. Flex sensors are attached to every finger and thumb of both hands so in a total of five flex sensors are sewed on each glove. MPU6050 (Accelerometer and Gyroscope) MPU6050 is a small size chip of 6 DOF as shown in figure 3 . It has both accelerometer and gyroscope built in. Rotation of hand is read from the accelerometer data while angular movement is tracked by the gyroscope. The data form both accelerometer and gyroscope are of 16 bits which are processed by the controller to detect the motion of hands 


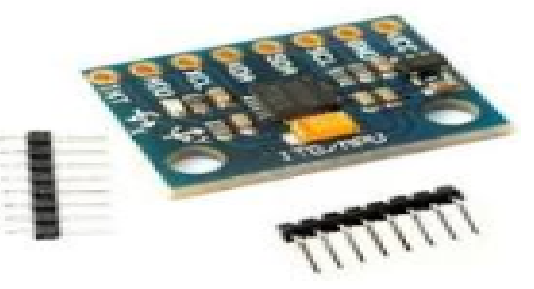

Fig.3: MPU6050 Accelerometer Sensor

\section{B. Signal Conditioning and Processing:}

Signal conditioning is required for the flex sensors to correctly obtain the data at desired voltage levels. Now, all the data from flex sensors and MPU6050 chip is processed in a microcontroller. The micro-controller selected is Arduino Pro Mini which is operated at $3.3 \mathrm{~V}$ at $8 \mathrm{MHz}$. It is a small size $0.7 \times 1.3$ " controller board, which makes the whole system portable and introduce higher mobility to the user. The controller board is based on ATMega328P micro-controller. Arduino Pro Mini is attached to the wrist of each hand to process the raw values from the gyroscope, accelerometer and flex sensors. The raw data is processed and transformed into the meaningful ones.

\section{Data Transmission and Reception:}

The processed data from one hand is multiplexed with the other via Bluetooth. HC-05 Bluetooth module is used for wireless communication because of its low price and high performance. The data receiving controller will also act as a master controller. This Bluetooth module is interfaced with the Arduino Pro Mini through UART pins (Tx, Rx) and operates at $3.3 \mathrm{~V}$. Bluetooth modules were configured so that no other Bluetooth module connects to them. Both Receiver and Transmitter Bluetooth was bind using AT commands. While gesturing, the data from the slave gets transferred to the master for further processing and recognition of the gesture recorded during the experiment. The data were saved in text MS Excel Sheet for the processing and easy visualization through the graph. Table 1 indicates the statement which is assigned for the corresponding finger of hand.

Table:1 Text assignment table

\begin{tabular}{|c|c|c|}
\hline Fingers & Value 1 & Text to be print \\
\hline Thumb & 1006 & Glad you like that . \\
\hline \multirow[t]{4}{*}{ Index } & 1016 & Super \\
\hline & $\begin{array}{l}1007- \\
1009\end{array}$ & Hi... \\
\hline & 1003 & Myself: I'm Jaikumar \\
\hline & 1005 & Keep moving man \\
\hline \multirow[t]{3}{*}{ Middle } & $\begin{array}{l}1013- \\
1016\end{array}$ & Salute \\
\hline & 1019 & $\begin{array}{l}\text { "Coimbatore Institute of } \\
\text { Technology" College name }\end{array}$ \\
\hline & 1013 & Hmm... I'm thinking \\
\hline
\end{tabular}

\section{Data Transceiver Module:}

The data transceiver module used in this work is HC-05 module, it is an easy to use Bluetooth Serial Port Protocol module, designed for transparent wireless serial connection setup. This serial connected port bluetooth module is fully qualified Bluetooth V2. It has an enhanced Data Rate of $3 \mathrm{Mbps}$ Modulation with complete $2.4 \mathrm{GHz}$ radio transceiver and baseband.

\section{E. Audio Model:}

Audio transmission display in android mobile/smart phone shows in fig 4.

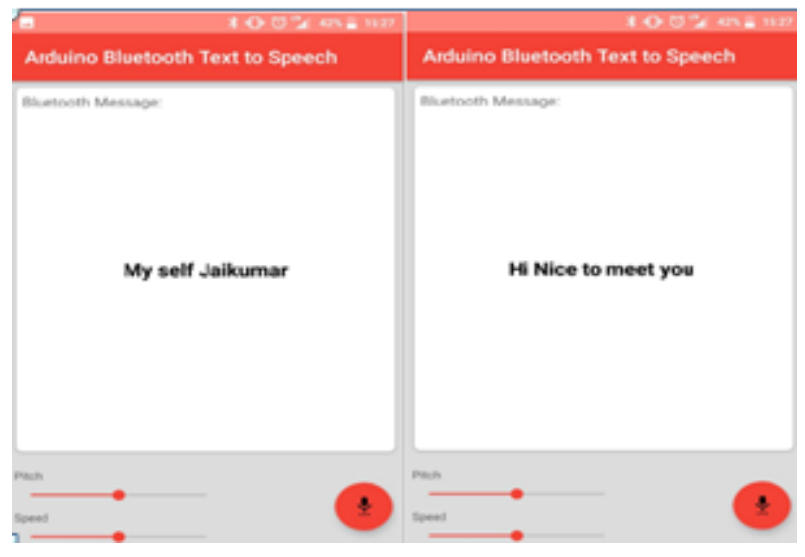

Fig.4: Audio output

\section{METHODOLOGY}

\section{A. Flexion}

As stated in the sections above, flex sensors and accelerometer are used to record the bending of fingers and orientation of hand respectively. Flex sensors change its resistance when it bends. This variation is changed into voltages through voltage divider configuration and further digitized with the ADC of the microcontroller. The ADC is of 10bit resolution. It will map input voltage from 0 to 3.3 volts into integer 1 to 1023 . The increase and decrease in the 10bit value points depend on the increase and decrease in the resistance of flex sensor. By doing a simple calculation, the integer value can be converted into the actual voltage. The values are further normalized between $[0,1]$ for easier analysis.

\section{B. Orientation of hands}

The orientation of hand is derived from the accelerometer and gyroscope data. The accelerometer and gyroscope module are interfaced with the controller and its raw values are obtained from its buffers and then processed by the controller to get the information of orientation of hands. The range of Accelerometer and Gyro is selected to be $+/-2 \mathrm{~g}$ and $+/$ $250 \% \mathrm{sec}$ respectively. The values are then scaled between -1 to +1 as (2) and (3).

\section{Gesture Mapping Sensors}

Data from both hands are multiplexed through Bluetooth modules. All the flex sensors data are converted into decimal values according to each gesture. For the similar values of both hands, data from accelerometer the gestures. Average values of each sensor are computed and matched with the gesture made. For similar values, accelerometer and gyroscope values are taken into the account. Gesture recognition is performed via lookup data. 


\section{Flow Diagram of the System}

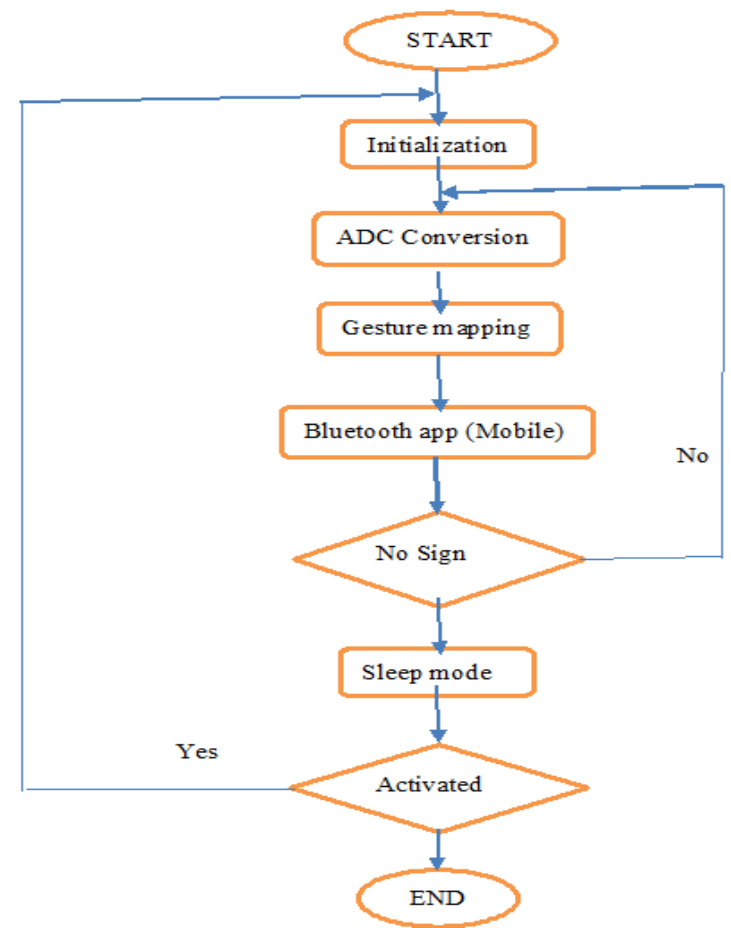

Fig 5: Flow table for gesture mapping

Flow diagram for gesture mapping is shown in figure 5. Flex sensors are stitched in all the finger slots of the gloves. The gloves can be worn by any deaf and dumb people and based on their hand movements an initial analog signal is created and then its digital equivalent is found out to map its gesture with the corresponding text. The text is made visualized for the people who is interacting with the deaf and dumb for better understanding and communication of the message. If the sign is not received the system waits for the sign to be rendered by the deaf and dumb.

\section{RESULTS}

A. Gesture Voice output:

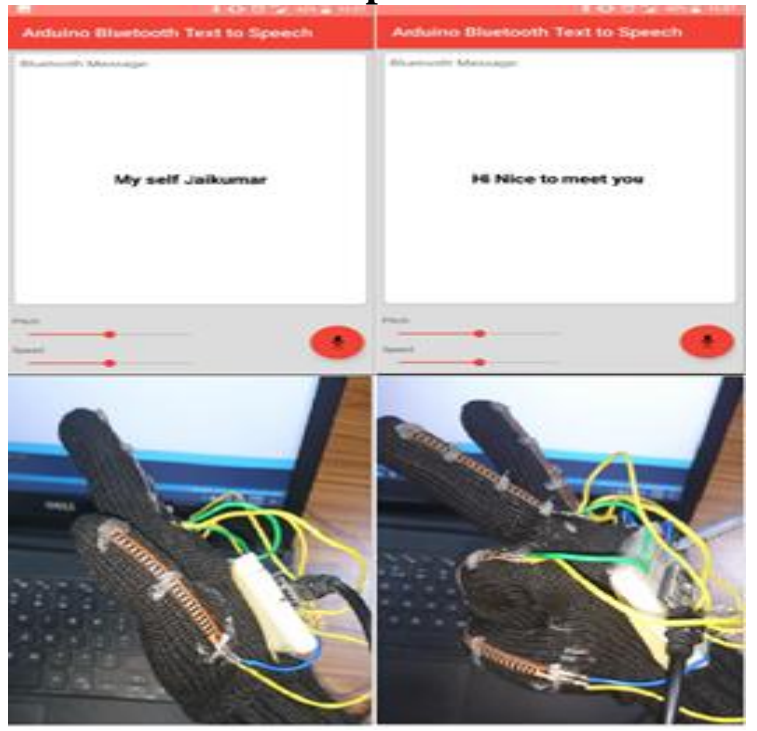

Fig. 6a: Gesture test for "self-introduction" b. Gesture test for "welcome Wish"
When a deaf and dumb person wants to introduce himself, the hand expression shown in figure $6 \mathrm{a}$ can be used to retrieve his/her name from the database and it is displayed in the text box of the recipient. Similarly figure $6 \mathrm{~b}$ shows a greeting message for the recipient.

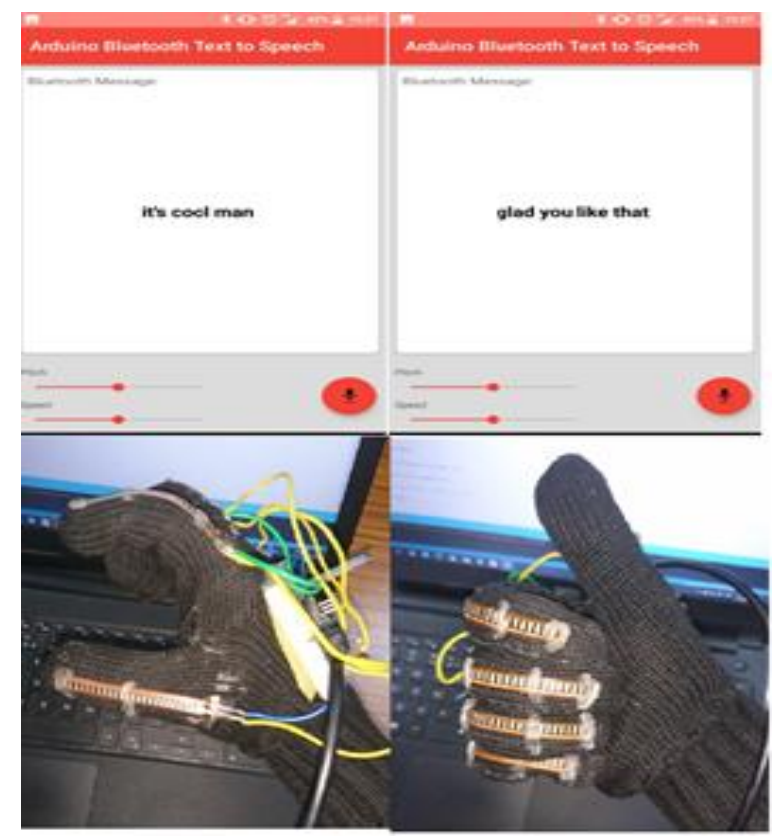

Fig. 7a: Gesture test for "perfect" b. Gesture test for "Likes"

Figure $7 \mathrm{a}$ and $7 \mathrm{~b}$ shows the gesture test result for intimating that what is happening now is perfect and like message. The efficiency of the system with the flexion values is $92 \%$ for the corresponding gestures in order to make a lookup table. The system was implemented and tested with the sample gestures it depends on the sign of language and the words were customized based on the flex values.

\section{B. Gesture Control Wheel chair}

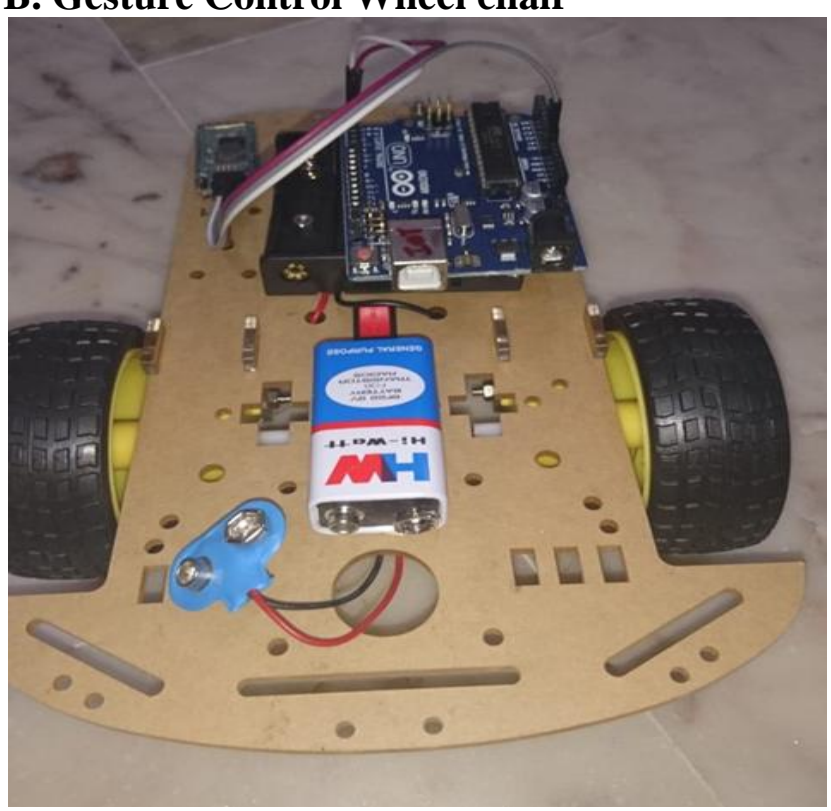

Fig 8: Back view of Wheel chair control 


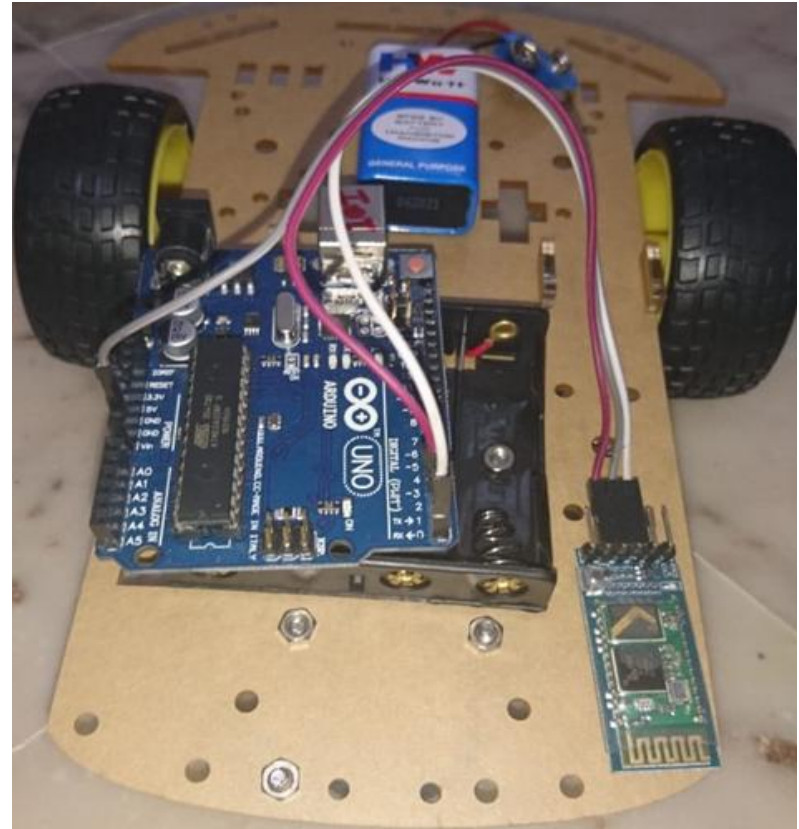

Fig 9: Front view Wheel chair control

In this work, a robotic wheel chair is also designed to help those who is both deaf and dumb person and physically handicapped and cannot move with their legs. Based on the hand gesture provided by the deaf and dumb person, the wheel chair moves forward and backwards to satisfy the need of the person. Fig 8 and 9 shows the back view and front view of the gesture control wheel chair. It consists of battery, wheels and the wheel chair is programmed using a Arduino UNO based controller as shown in figures.

\section{CONCLUSION}

The proposed system translates the India Sign Language to speech with the help of various sensors such that the flex sensors, accelerometer, gyroscope and contact sensor. The system achieved the efficiency of $93.4 \%$ with alphabets and numbers and is expected to vary with more gestures and database. The wireless communication and small size modules make it an autonomous, portable and a user-friendly system. Keeping in view, the system is made from cheap and inexpensive modules without compromising the system performance. It can be modified to the extensive vocabulary of Sign Language so that the deaf community can easily communicate with other people and controlling the wheel chair.

\section{ACKNOWLEDGMENTS}

Our thanks to the experts who have contributed towards development of the work.

\section{REFERENCES}

[1] B.G Lee and S.M Lee, "Smart Wearable Hand Device for Sign Language Interpretation System With Sensors Fusion", IEEE Sensor Journal, Vol.18. No. 3, pp.no: 1224-1232, September 2018.

[2] Chun Zhu and Weihua Sheng, "Wearable Sensor-Based Hand Gesture and Daily Activity Recognition for RobotAssisted Living", IEEE transactions on systems, man, and cybernetics, 1083-4427, pp.no: 1-5, 2017.

[3] S.V.Matiwade and M.R.Dixit, "Electronics Voice for deaf and dumb people using flex sensors", International Journal of Engineering Research and Reviews ISSN 2348-697X (Online), Vol. 7, no. 2, pp: 27-32, April June 2019.

[4] Mikhail G. Grif, Alexey L. Prikhodko Novosibirsk, "Approach to The Sign Language Gesture Recognition Framework Based on HamNoSys Analysis", XIV Международная научно-техническая конференция АПЭП, 978-1-5386-7054-5/18, pp.no: 426- 429, 2018.

[5] Hardik Rewari, Vishal Dixit, Dhroov Batra, Hema N, "Automated Sign Language Interpreter", IEEE Explore Proceedings of 2018 Eleventh International Conference on Contemporary Computing (IC3), 2-4 978-1-53866835-1/18, 2018.

[6] Andrews Samraj, Naser Mehrdel and Shohel Sayeed "Sign language communication and authentication using sensor fusion of hand glove and photometric signals", IEEE Explore 8th International Conference on Information Technology (ICIT), 978-1-5090-6332-1/17, pp.no: 214-219, 2017.

[7] Soniya D. Makwana; Anuradha G. Tandon, "Touch screen based wireless multifunctional wheelchair using ARM and PIC microcontroller", 2016 International Conference on Microelectronics, Computing and Communications(MicroCom),doi:10.1109/microcom.201 $6.7522524,2016$.

[8] Vishal P. Bhujbal, K.K. Warhade, "Hand sign recognition based communication system for speech disable people", Second International Conference on Intelligent Computing and Control Systems (ICICCS), 10.1109/ICCONS.2018.8663054, pp.no: 348-352, March 2019. 